\title{
Algunas observaciones sobre la durabilidad frente a los sulfatos del mortero de cemento portland, carbonatado y sin carbonatar. Estudio por espectroscopía infrarroja
}

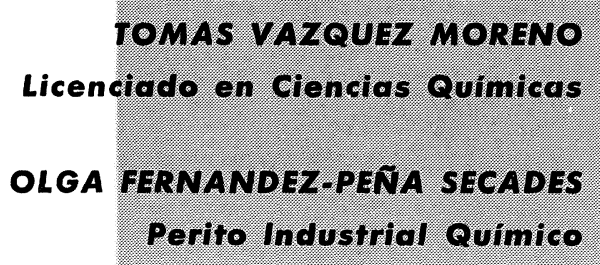

Al objeto de poder comprobar y comparar los resultados obtenidos sobre una serie de ensayos de prismas pequeños (KOCH y STEINEGGER) de $1 \times 1 \times 6 \mathrm{~cm}$, hechos en colaboración con el "Subcomité de Resistencia Química" del CEMBUREAU, en el I.E.T.c.c. se realizaron tres nuevas series de ensayo con ocho cementos distintos, una de las cuales nos sirvió para estudiar el efecto inhibidor de la carbonatación.

También sobre dos de estas series se ha efectuado un estudio de espectroscopía infrarroja, para un mejor esclarecimiento de la durabilidad del cemento portland frente a los sulfatos.

En este artículo se resumen los resultados obtenidos, cuyo estudio completo saldrá en una próxima publicación del I.E.T.c.c. 


\section{CEMENTO EMPleado: CeMENTo TIPO PORTLAND; CATEgORIA P-450}

Análisis de la muestra:

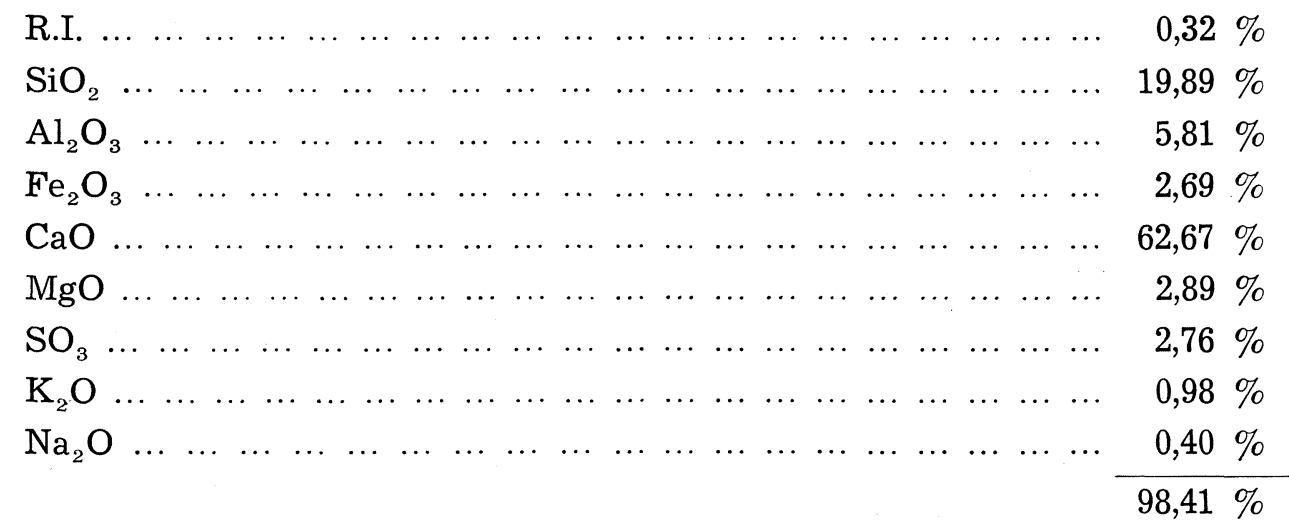

$\mathrm{CaO}$ libre $=0,92 \%$.

\section{DISOLUCIONES AGRESIVAS EMPLEADAS}

- Agua filtrada, para la conservación de los prismas testigo.

- Disolución de sulfato cálcico a saturación.

- Disolución mezcla de sulfatos cálcico y magnésico al $50 \%$, y de igual concentración.

- Agua de mar artificial.

-- Disolución de sulfato sódico al $5 \%$.

- Disolución de sulfato magnésico al $10 \%$.

- Disolución de sulfato amónico al $5 \%$.

- Disolución de cloruro amónico al $5 \%$.

\section{ENSAYOS REALIZADOS}

Una vez confeccionados los prismas se curaron en cámara de humedad (h.r. $=100 \%$ ) durante 28 días. Inmediatamente después del curado previo se colocaron los prismas en las disoluciones agresivas (10 prismas por disolución y por edad estudiadas), hasta el momento de la determinación de la resistencia a flexotracción.

Las edades estudiadas fueron $1,3,6,9$ y 12 meses. Como valores individuales de rotura se tomaron las medias aritméticas de los diez prismas ensayados. Estos valores de rotura se han comparado con los valores de rotura obtenidos sobre los prismas testigo a la misma edad, dividiendo la media aritmética de las resistencias alcanzadas por los prismas ensayados, entre la media aritmética de las resistencias alcanzadas por los prismas testigo a la misma edad. El coeficiente obtenido es el denominado "índice de durabilidad" o "coeficiente de corrosión por sulfatos".

Los resultados obtenidos se dan en los cuadros: 1, 1a; 2, 2a; 3 , 3a y 4, que figuran a continuación. 
C U A D R O 1

Cemento: P-450.- Resistencias a flexotracción; datos estadísticos de los resultados obtenidos $\overline{\mathrm{X}}=$ media aritmética $; \mathrm{S}=\frac{\left(\mathrm{X}_{2}-\overline{\mathrm{X}}\right)^{2}}{\mathrm{~N}-1}=$ desviación tipo $; \mathrm{V}=\frac{\mathrm{S} \cdot 100}{\overline{\mathrm{X}}}=$ coeficiente de variación

\begin{tabular}{|c|c|c|c|c|c|c|c|c|c|c|c|c|}
\hline \multirow{2}{*}{$\begin{array}{c}\text { Edad } \\
\text { (meses) }\end{array}$} & \multicolumn{3}{|c|}{ Agua filtrada } & \multicolumn{3}{|c|}{$\begin{array}{c}\text { Disolución de } \\
\text { so }_{4} \mathrm{Ca} \text { a saturación }\end{array}$} & \multicolumn{3}{|c|}{$\begin{array}{c}\text { Mezcla al 50 \% de } \\
\text { disolución de } \\
\mathrm{SO}_{4} \mathrm{Ca}+\mathrm{SO}_{4} \mathrm{Mg}\end{array}$} & \multicolumn{3}{|c|}{ Agua de mar artificial } \\
\hline & $\overline{\mathbf{x}}$ & $s$ & $\mathrm{v}$ & $\overline{\mathbf{x}}$ & $s$ & $\mathrm{v}$ & $\overline{\mathbf{x}}$ & $s$ & $\mathbf{v}$ & $\overline{\mathbf{x}}$ & $s$ & $\mathrm{v}$ \\
\hline 1 & 81,77 & 6,16 & 7,53 & 86,46 & 5,58 & 6,46 & 87,95 & 7,08 & 8,05 & 82,79 & 7,62 & 9,21 \\
\hline 3 & 84,36 & 3,80 & 4,00 & 93,22 & 7,38 & 7,91 & 94,18 & 6,06 & 6,44 & 69,79 & 5,64 & 8,89 \\
\hline 6 & 86,00 & 4,66 & 5,42 & 97,89 & 7,50 & 7,67 & 109,35 & 7,68 & 7,02 & 79,71 & 4,44 & 5,58 \\
\hline 9 & 86,28 & 5,87 & 6,80 & 105,90 & 8,21 & 7,76 & 97,24 & 12,66 & 13,02 & 82,61 & 6,45 & 7,81 \\
\hline 12 & 90,97 & 4,69 & 5,15 & 102,00 & 7,53 & 7,39 & 96,00 & 13,12 & 13,67 & 81,01 & 8,15 & 10,06 \\
\hline \multirow{2}{*}{$\begin{array}{c}\text { Edad } \\
\text { (meses) }\end{array}$} & \multicolumn{3}{|c|}{$\begin{array}{l}\text { Disolución de } \\
\mathrm{SO}_{4} \mathrm{Na}_{2} \text { al } 5 \%\end{array}$} & \multicolumn{3}{|c|}{$\begin{array}{l}\text { Disolución de } \\
\text { SO }_{4} \mathrm{Mg} \text { al } 10 \%\end{array}$} & \multicolumn{3}{|c|}{$\begin{aligned} & \text { Disolución de } \text { de } \\
& \mathbf{S O}_{4}\left(\mathrm{NH}_{4}\right)_{2} \text { al } 5 \%\end{aligned}$} & \multicolumn{3}{|c|}{$\begin{array}{l}\text { Disolución de } \\
\text { ClNH }_{4} \text { al } 5 \%\end{array}$} \\
\hline & $\overline{\mathbf{x}}$ & $s$ & V & $\overline{\mathbf{x}}$ & $S$ & $\mathrm{~V}$ & $\overline{\mathbf{x}}$ & $S$ & $\mathrm{v}$ & $\overline{\mathbf{x}}$ & $s$ & $\mathrm{~V}$ \\
\hline 1 & 100,65 & 3,56 & 3,56 & 100,90 & 8,38 & 8,30 & 14,61 & 4,79 & 32,83 & 47,55 & 4,70 & 9,89 \\
\hline 3 & 96,67 & 12,13 & 12,55 & 101,76 & 8,34 & 8,20 & 8,50 & 1,45 & 17,05 & 42,52 & 5,27 & 12,39 \\
\hline 6 & 94,26 & 16,04 & 17,02 & 88,90 & 8,93 & 10,04 & 12,19 & 2,83 & 23,62 & 39,67 & 5,54 & 13,97 \\
\hline 9 & 64,49 & 14,02 & 22,08 & 67,73 & 16,44 & 24,27 & 10,34 & 2,05 & 19,82 & 34,06 & 5,42 & 15,93 \\
\hline 12 & 36,28 & 16,59 & 45,74 & 35,28 & 4,43 & 12,57 & 10,10 & 2,01 & 20,00 & 31,69 & 8,67 & 27,38 \\
\hline
\end{tabular}

CuAdro 1a

Cemento: P-450.- Coeficientes de corrosión; datos estadísticos de los resultados obtenidos $\overline{\mathrm{X}}=$ media aritmética $; \mathrm{S}=\frac{\left(\mathrm{X}_{2}-\overline{\mathrm{X}}\right)^{2}}{\mathrm{~N}-1}=$ desviación tipo $\mathrm{V}=\frac{\mathrm{S} \cdot 100}{\overline{\mathrm{X}}}=$ coeficiente de variación

\begin{tabular}{|c|c|c|c|c|c|c|c|c|c|c|c|c|}
\hline \multirow{2}{*}{$\begin{array}{c}\text { Edad } \\
\text { (meses) }\end{array}$} & \multicolumn{3}{|c|}{$\begin{array}{l}\text { Disolución de } \\
\text { SO }_{4} \mathrm{Ca} \text { a saturación }\end{array}$} & \multicolumn{3}{|c|}{$\begin{array}{c}\text { Mezcla al 50 \% de } \\
\text { disolución de } \\
\mathrm{SO}_{4} \mathrm{Ca}+\mathrm{SO}_{4} \mathrm{Mg}\end{array}$} & \multicolumn{3}{|c|}{ Agua de mar artificial } & \multicolumn{3}{|c|}{$\begin{array}{l}\text { Disolución de } \\
\mathbf{S O}_{4} \mathbf{N a}_{2} \text { al } 5 \%\end{array}$} \\
\hline & $\overline{\mathbf{x}}$ & $s$ & $\mathrm{v}$ & $\overline{\mathbf{x}}$ & $s$ & $\mathrm{~V}$ & & $s$ & $\mathrm{v}$ & $\overline{\mathbf{x}}$ & $s$ & $\mathbf{v}$ \\
\hline 1 & 1,06 & 0,07 & 6,4 & 1,10 & 0,07 & 6,55 & & 0,07 & 7,32 & 1,23 & 0,04 & 3,54 \\
\hline 3 & 1,10 & 0,07 & 6,0 & 1,11 & 0,07 & 6,52 & & 0,07 & 8,11 & 1,16 & 0,38 & 33,10 \\
\hline 6 & 1,13 & 0,09 & 7,6 & 1,27 & 0,09 & 7,00 & & 0,05 & 5,50 & 1,09 & 0,18 & 17,01 \\
\hline 9 & 1,22 & 0,09 & 7,7 & 1,12 & 0,46 & 41,12 & & 0,06 & 6,11 & 0,75 & 0,16 & 22,06 \\
\hline 12 & 1,12 & 0,06 & 5,7 & 1,06 & 0,14 & 13,77 & & 0,09 & 10,04 & 0,39 & 0,15 & 37,94 \\
\hline \multirow{2}{*}{$\begin{array}{l}\text { Edad } \\
\text { (meses) }\end{array}$} & \multicolumn{4}{|c|}{$\begin{array}{l}\text { Disolución de } \\
\text { SO }_{4} \mathrm{Mg} \text { al } 10 \%\end{array}$} & \multicolumn{4}{|c|}{$\begin{array}{c}\text { Disolución de } \\
\text { SO }_{4}\left(\mathbf{N H}_{4}\right)_{2} \text { al } 5 \%\end{array}$} & \multicolumn{4}{|c|}{$\begin{array}{l}\text { Disolución } \\
\text { CINH }_{4} \text { al } \\
5\end{array}$} \\
\hline & $\overline{\mathbf{x}}$ & & $s$ & $\mathrm{v}$ & $\overline{\mathbf{x}}$ & & $s$ & $\mathbf{v}$ & $\overline{\mathbf{x}}$ & $s$ & & $\mathrm{v}$ \\
\hline 1 & 1,23 & & 0,10 & 8,34 & 0,17 & & 0,06 & 35,29 & 0,58 & 0,06 & & 10,80 \\
\hline 3 & 1,20 & & 0,12 & 10,14 & 0,11 & & 0,02 & 17,20 & 0,50 & 0,06 & & 13,10 \\
\hline 6 & 1,03 & & 0,10 & 10,14 & 0,14 & & 0,03 & 21,12 & 0,46 & 0,06 & & 14,00 \\
\hline 9 & 0,73 & & 0,16 & 20,65 & 0,12 & & 0,02 & 18,63 & 0,39 & 0,06 & & 16,10 \\
\hline 12 & 0,39 & & 0,04 & 12,56 & 0,11 & & 0,02 & 20,13 & 0,35 & 0,09 & & 25,55 \\
\hline
\end{tabular}


C UADRO 2

Cemento: P-450.- Resistencias a flexotracción; datos estadísticos de los resultados obtenidos $\overline{\mathrm{X}}=$ media aritmética; $\mathrm{S}=\frac{\left(\mathrm{X}_{2}-\overline{\mathrm{X}}\right)^{2}}{\mathrm{~N}-1}=$ desviación tipo; $\mathrm{V}=\frac{\mathrm{S} \cdot 100}{\overline{\mathrm{X}}}=$ coeficiente de variación

\begin{tabular}{|c|c|c|c|c|c|c|c|c|c|c|c|c|}
\hline \multirow{2}{*}{$\begin{array}{c}\text { Edad } \\
\text { (meses) }\end{array}$} & \multicolumn{3}{|c|}{ Agua filtrada } & \multicolumn{3}{|c|}{$\begin{array}{l}\text { Disolución de } \\
\text { SO }_{4} \mathrm{Ca} \text { a saturación }\end{array}$} & \multicolumn{3}{|c|}{$\begin{array}{l}\text { Mezcla al 50 \% de } \\
\text { disolución de } \\
\mathrm{SO}_{4} \mathrm{Ca}+\mathrm{SO}_{4} \mathrm{Mg}\end{array}$} & \multicolumn{3}{|c|}{ Agua de mar artificial } \\
\hline & $\overline{\mathbf{x}}$ & $s$ & $\mathbf{v}$ & $\overline{\mathbf{x}}$ & $s$ & $\mathbf{v}$ & $\overline{\mathbf{x}}$ & $s$ & $\mathbf{v}$ & $\overline{\mathbf{x}}$ & $s$ & $\mathbf{v}$ \\
\hline 1 & 99,44 & 3,53 & 3,55 & 107,69 & 31,30 & 29,06 & 104,33 & 4,13 & 3,69 & 92,64 & 3,11 & 3,35 \\
\hline 3 & 104,89 & 2,41 & 2,30 & 109,64 & 4,68 & 4,27 & 106,75 & 4,19 & 3,92 & 95,64 & 4,54 & 4,75 \\
\hline 6 & 102,62 & 4,16 & 4,06 & 112,34 & 3,65 & 3,25 & 114,22 & 4,83 & 4,23 & 99,22 & 4,73 & 4,77 \\
\hline 9 & 100,81 & 5,10 & 5,06 & 110,00 & 3,93 & 3,57 & 102,76 & 4,68 & 4,55 & 100,64 & 3,67 & 3,65 \\
\hline 12 & 94,67 & 3,69 & 3,90 & 109,54 & 3,13 & 2,85 & 111,59 & 4,81 & 4,31 & 95,00 & 4,53 & 4,77 \\
\hline \multirow{2}{*}{$\begin{array}{c}\text { Edad } \\
\text { (meses) }\end{array}$} & \multicolumn{3}{|c|}{$\begin{array}{l}\text { Disolución de } \\
\mathrm{SO}_{4} \mathrm{Na}_{2} \text { al } 5 \%\end{array}$} & \multicolumn{3}{|c|}{$\begin{array}{l}\text { Disolución de } \\
\text { SO}_{4} \mathrm{Mg} \text { al } 10 \%\end{array}$} & \multicolumn{3}{|c|}{$\begin{array}{c}\text { Disolución de } \\
\mathrm{SO}_{4}\left(\mathrm{NH}_{4}\right)_{2} \text { al } 5 \%\end{array}$} & \multicolumn{3}{|c|}{$\begin{array}{l}\text { Disolución de } \\
\text { CINH }_{4} \text { al } 5 \quad \%\end{array}$} \\
\hline & $\overline{\mathbf{x}}$ & $s$ & $\mathrm{v}$ & $\overline{\mathbf{x}}$ & $\mathbf{s}$ & $\mathbf{v}$ & $\overline{\mathbf{x}}$ & $S$ & $\mathrm{v}$ & $\overline{\mathbf{x}}$ & $s$ & V \\
\hline 1 & 109,63 & 4,80 & 4,38 & 99,74 & 3,94 & 3,95 & 18,44 & 6,75 & 36,64 & 71,38 & 4,43 & 6,20 \\
\hline 3 & 106,00 & 4,48 & 4,22 & 106,00 & 4,05 & 3,79 & 7,20 & 2,35 & 32,74 & 58,73 & 4,01 & 6,83 \\
\hline 6 & 112,46 & 2,70 & 2,40 & 107,36 & 2,07 & 1,93 & 13,25 & 5,09 & 38,42 & 44,19 & 3,55 & 8,03 \\
\hline 9 & 57,62 & 30,91 & 53,65 & 55,00 & 22,04 & 40,13 & 6,36 & 3,84 & 60,43 & 40,75 & 3,63 & 8,92 \\
\hline 12 & 53,92 & 38,75 & 71,88 & 40,97 & 6,31 & 15,42 & 10,38 & 4,22 & 40,65 & 32,26 & 2,87 & 8,89 \\
\hline
\end{tabular}

C U A D R O $2 \mathrm{a}$

Cemento: P-450.- Coeficientes de corrosión; datos estadísticos de los resultados obtenidos $\overline{\mathrm{X}}=$ media aritmética $; \mathrm{S}=\frac{\left(\mathrm{X}_{2}-\overline{\mathrm{X}}\right)^{2}}{\mathrm{~N}-1}=$ desviación tipo $; \mathrm{V}=\frac{\mathrm{S} \cdot 100}{\overline{\mathrm{X}}}=$ coeficiente de variación

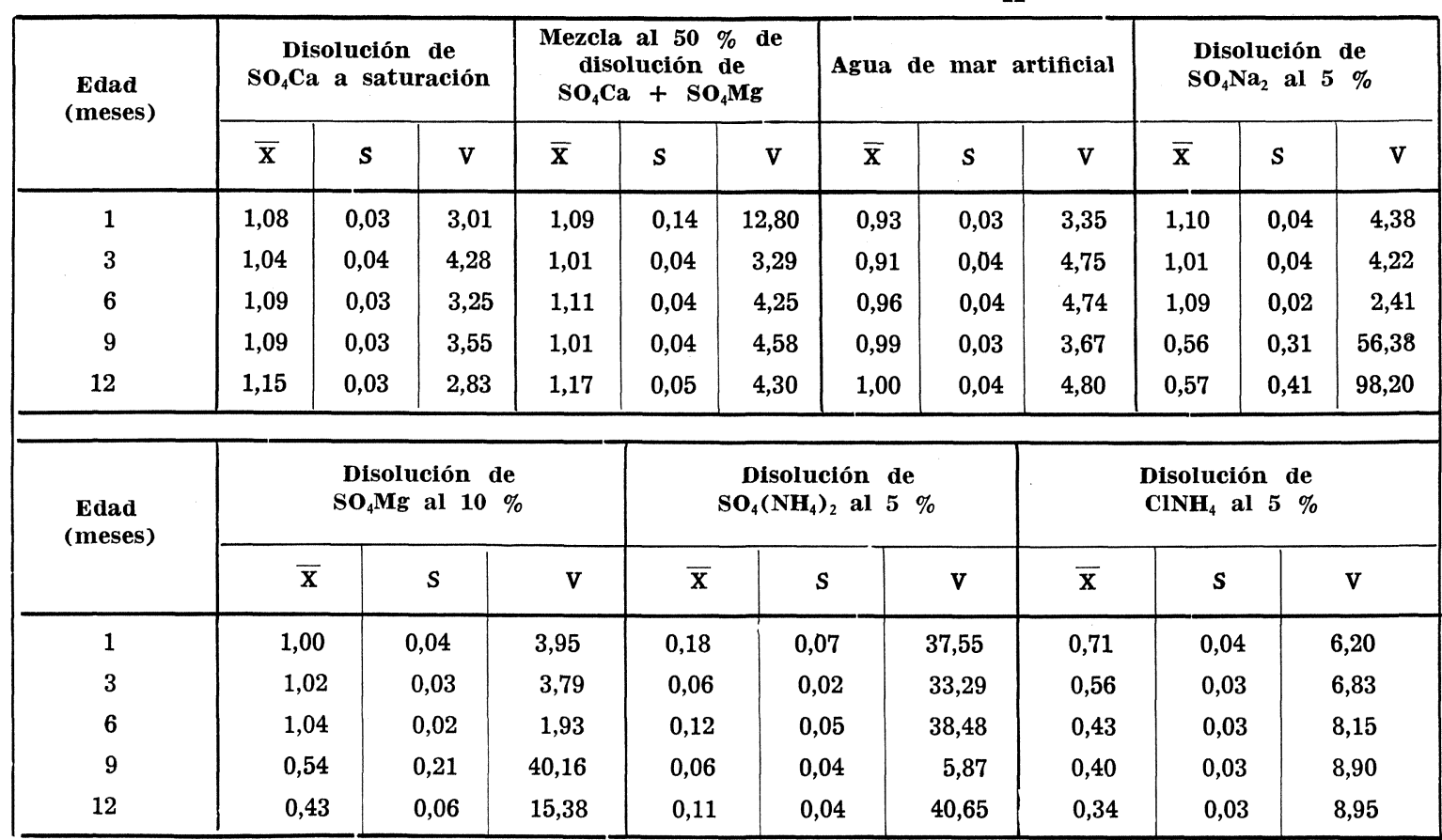


CUADRO 3

Cemento: P-450.- Resistencias a flexotracción; datos estadísticos de los resultados obtenidos $\overline{\mathrm{X}}=$ media aritmética $; \mathrm{S}=\frac{\left(\mathrm{X}_{2}-\overline{\mathrm{X}}\right)^{2}}{\mathrm{~N}-1}=$ desviación tipo; $\mathrm{V}=\frac{\mathrm{S} \cdot 100}{\overline{\mathrm{X}}}=$ coeficiente de variación

\begin{tabular}{|c|c|c|c|c|c|c|c|c|c|c|c|c|}
\hline \multirow{2}{*}{$\begin{array}{c}\text { Edad } \\
\text { (meses) }\end{array}$} & \multicolumn{3}{|c|}{ Agua filtrada } & \multicolumn{3}{|c|}{$\begin{array}{c}\text { Disolución de } \\
\text { SO }_{4} \text { Ca a saturación }\end{array}$} & \multicolumn{3}{|c|}{$\begin{array}{l}\text { Mezcla al 50\% de } \\
\text { disolución de } \\
\mathrm{SO}_{4} \mathrm{Ca}+\mathrm{SO}_{4} \mathrm{Mg}\end{array}$} & \multicolumn{3}{|c|}{ Agua de mar artificial } \\
\hline & $\overline{\mathbf{x}}$ & $s$ & $\mathrm{v}$ & $\overline{\mathbf{x}}$ & $s$ & $\mathbf{v}$ & $\overline{\mathbf{x}}$ & $s$ & $\mathbf{v}$ & $\overline{\mathbf{x}}$ & $s$ & $\mathbf{v}$ \\
\hline 1 & 99,46 & 3,23 & 3,25 & 113,76 & 4,06 & 3,57 & 104,99 & 4,34 & 4,14 & 90,32 & 3,94 & 4,36 \\
\hline 3 & 104,52 & 2,88 & 2,75 & 111,01 & 4,16 & 3,75 & 112,67 & 3,34 & 2,96 & 95,43 & 2,62 & 2,74 \\
\hline 6 & 100,82 & 2,95 & 2,93 & 120,36 & 2,05 & 1,71 & 118,50 & 4,81 & 4,06 & 96,35 & 5,18 & 5,38 \\
\hline 9 & 102,15 & 3,50 & 3,43 & 110,11 & 4,73 & 4,29 & 97,13 & 4,44 & 4,57 & 83,11 & 4,52 & 5,44 \\
\hline 12 & 101,86 & 4,10 & 4,02 & 110,31 & 4,33 & 3,92 & 101,35 & 5,04 & 4,98 & 91,81 & 3,84 & 4,18 \\
\hline \multirow{2}{*}{$\begin{array}{c}\text { Edad } \\
\text { (meses) }\end{array}$} & \multicolumn{3}{|c|}{$\begin{array}{l}\text { Disolución de } \\
\mathrm{SO}_{4} \mathrm{Na}_{2} \text { al } 5 \%\end{array}$} & \multicolumn{3}{|c|}{$\begin{array}{l}\text { Disolución de } \\
\text { SO }{ }_{4} \mathbf{M g} \text { al } 10 \%\end{array}$} & \multicolumn{3}{|c|}{ 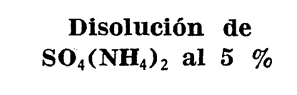 } & \multicolumn{3}{|c|}{$\begin{array}{l}\text { Disolución de } \\
\text { ClNH }_{4} \text { al } 5 \%\end{array}$} \\
\hline & $\overline{\mathrm{x}}$ & $S$ & $\mathbf{v}$ & $\overline{\mathbf{x}}$ & $s$ & $\mathrm{v}$ & $\overline{\mathbf{x}}$ & $s$ & $\mathbf{v}$ & $\overline{\mathbf{x}}$ & $s$ & $\mathbf{v}$ \\
\hline 1 & 115,10 & 3,38 & 2,94 & 111,09 & 2,76 & 2,48 & 26,36 & 6,26 & 23,76 & 65,81 & 5,71 & 8,67 \\
\hline 3 & 106,06 & 6,84 & 6,33 & 103,74 & 3,52 & 3,40 & 4,94 & 1,63 & 23,52 & 58,95 & 3,33 & 5,66 \\
\hline 6 & 107,02 & 8,80 & 8,22 & 89,50 & 4,86 & 5,43 & 10,19 & 3,06 & 30,08 & 54,34 & 7,73 & 14,22 \\
\hline 9 & 60,41 & 12,55 & 20,77 & 91,20 & 4,71 & 5,16 & 12,73 & 3,25 & 25,55 & 58,35 & 8,44 & 14,47 \\
\hline 12 & $*$ & $*$ & $*$ & 37,15 & 10,28 & 27,64 & 11,41 & 15,14 & 32,75 & 35,79 & 7,00 & 19,58 \\
\hline
\end{tabular}

* No pudieron medirse por estar desintegradas.

C U A D R o $3 \mathrm{a}$

Cemento: P-450.- Coeficientes de corrosión; datos estadísticos de los resultados obtenidos $\overline{\mathrm{X}}=$ media aritmética; $\mathrm{S}=\frac{\left(\mathrm{X}_{2}-\overline{\mathrm{X}}\right)^{2}}{\mathrm{~N}-1}=$ desviación tipo; $\mathrm{V}=\frac{\mathrm{S} \cdot 100}{\overline{\mathrm{X}}}=$ coeficiente de variación

\begin{tabular}{|c|c|c|c|c|c|c|c|c|c|c|c|c|}
\hline \multirow{2}{*}{$\begin{array}{c}\text { Edad } \\
\text { (meses) }\end{array}$} & \multicolumn{3}{|c|}{$\begin{array}{l}\text { Disolución de } \\
\mathrm{SO}_{4} \mathrm{Ca} \text { a saturaciór }\end{array}$} & \multicolumn{3}{|c|}{$\begin{array}{c}\text { Mezcla al 50 \% de } \\
\text { disolución de } \\
\mathrm{SO}_{4} \mathrm{Ca}+\mathrm{SO}_{4} \mathrm{Mg}\end{array}$} & \multicolumn{3}{|c|}{ Agua de mar artificial } & \multicolumn{3}{|c|}{$\begin{array}{l}\text { Disolución de } \\
\mathrm{SO}_{4} \mathrm{Na}_{2} \text { al } 5 \quad \%\end{array}$} \\
\hline & $\overline{\mathbf{x}}$ & $\mathbf{S}$ & & $\overline{\mathbf{x}}$ & $s$ & $\mathrm{v}$ & & $s$ & $\mathrm{v}$ & $\overline{\mathbf{x}}$ & $s$ & $\mathbf{v}$ \\
\hline 1 & 1,14 & 0,04 & & 1,05 & 0,04 & 4,14 & & 0,04 & 42,95 & 1,16 & 0,03 & 2,93 \\
\hline 3 & 1,06 & 0,04 & & 1,07 & 0,03 & 2,96 & & 0,02 & 2,74 & 1,03 & 0,06 & 6,33 \\
\hline 6 & 1,19 & 0,02 & & 1,17 & 0,04 & 4,06 & & 0,51 & 5,38 & 1,06 & 0,08 & 8,22 \\
\hline 9 & 1,07 & 0,04 & 4 & 0,95 & 0,04 & 4,57 & & 0,04 & 5,44 & 0,59 & 0,12 & 20,77 \\
\hline 12 & 1,08 & 0,04 & & 0,99 & 0,05 & 4,98 & & 0,03 & 4,22 & * & * & $*$ \\
\hline \multirow{2}{*}{$\begin{array}{c}\text { Edad } \\
\text { (meses) }\end{array}$} & \multicolumn{4}{|c|}{$\begin{array}{l}\text { Disolución de } \\
\text { SO }_{4} \mathrm{Mg} \text { al } 10 \%\end{array}$} & \multicolumn{4}{|c|}{$\begin{array}{cl}\text { Disolución de } & \text { de } \\
\mathbf{S O}_{4}\left(\mathrm{NH}_{4}\right)_{2} & \text { al } 5 \%\end{array}$} & \multicolumn{4}{|c|}{$\begin{array}{l}\text { Disolución de } \\
\text { CINH }_{4} \text { al } 5 \%\end{array}$} \\
\hline & $\overline{\mathbf{x}}$ & \multicolumn{2}{|r|}{$s$} & $\mathbf{v}$ & $\overline{\mathbf{x}}$ & \multicolumn{2}{|c|}{$s$} & $\mathbf{v}$ & $\overline{\mathbf{x}}$ & \multicolumn{2}{|l|}{$s$} & $\mathbf{v}$ \\
\hline 1 & 1,11 & \multicolumn{2}{|c|}{0,03} & 2,48 & 0,26 & \multicolumn{2}{|c|}{0,06} & 23,76 & 0,66 & \multicolumn{2}{|c|}{0,05} & 8,67 \\
\hline 3 & 0,99 & \multicolumn{2}{|c|}{0,03} & 3,40 & 0,06 & \multicolumn{2}{|c|}{0,01} & 23,50 & 0,56 & \multicolumn{2}{|c|}{0,03} & 5,66 \\
\hline 6 & 0,88 & \multicolumn{2}{|c|}{0,05} & 5,43 & 0,10 & \multicolumn{2}{|c|}{0,03} & 3,09 & 0,54 & \multicolumn{2}{|c|}{0,07} & 14,23 \\
\hline 9 & 0,89 & \multicolumn{2}{|c|}{0,04} & 5,16 & 0,12 & \multicolumn{2}{|c|}{0,03} & 25,55 & 0,57 & \multicolumn{2}{|c|}{0,08} & 14,77 \\
\hline 12 & 0,36 & & 0,10 & 27,67 & 0,11 & & & 132,80 & 0,35 & $0,0^{\prime}$ & & 9,59 \\
\hline
\end{tabular}

* No pudieron medirse por estar desintegradas. 


\section{UADRO 4}

Comparación de las velocidades de disolución entre los prismas sin carbonatar y los prismas carbonatados

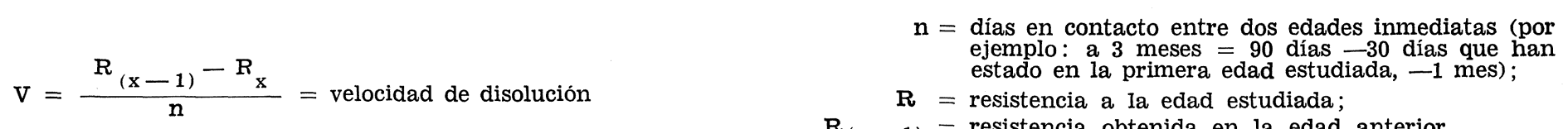

$\mathbf{R}=$ resistencia a la edad estudiada;

$R_{(x-1)}=$ resistencia obtenida en la edad anterior.

\begin{tabular}{|c|c|c|c|c|c|c|c|c|c|}
\hline \multirow[t]{3}{*}{$\begin{array}{c}\text { Edad } \\
\text { (meses) }\end{array}$} & \multirow[t]{3}{*}{$\begin{array}{c}\text { Valor de } \\
\text { n }\end{array}$} & \multicolumn{2}{|c|}{ Agua filtrada } & \multicolumn{2}{|c|}{$\begin{array}{c}\text { Disolución de } \\
\mathrm{SO}_{4} \mathrm{Ca} \text { a saturación }\end{array}$} & \multicolumn{2}{|c|}{$\begin{array}{c}\text { Mezcla al 50 \% de } \\
\text { disolución de } \\
\mathbf{S O}_{4} \mathbf{C a}+\mathbf{S O}_{4} \mathbf{M g}\end{array}$} & \multicolumn{2}{|c|}{ Agua de mar artificial } \\
\hline & & \multicolumn{2}{|c|}{ Velocidad de disolución } & \multicolumn{2}{|c|}{ Velocidad de disolución } & \multicolumn{2}{|c|}{ Velocidad de disolución } & \multicolumn{2}{|c|}{ Velocidad de disolución } \\
\hline & & Sin carbonatar & Carbonatados & Sin carbonatar & Carbonatados & Sin carbonatar & Carbonatados & Sin carbonatar & Carbonatados \\
\hline 3 & $\mathrm{n}=60$ & $9,1 \times 10^{-2}$ & $-8,4 \times 10^{-2}$ & $-3,2 \times 10^{-2}$ & $-4,6 \times 10^{-2}$ & $-4,0 \times 10^{-2}$ & $-1,3 \times 10^{-1}$ & $-5,0 \times 10^{-2}$ & $-8,5 \times 10^{-2}$ \\
\hline 6 & $\mathrm{n}=90$ & $2,5 \times 10^{-2}$ & $4,1 \times 10^{-2}$ & $-3,0 \times 10^{-2}$ & $-1,0 \times 10^{-1}$ & $-8,3 \times 10^{-2}$ & $-6,5 \times 10^{-2}$ & $-4,0 \times 10^{-2}$ & $-1,0 \times 10^{-}$ \\
\hline 9 & $\mathrm{n}=90$ & $2,0 \times 10^{-2}$ & $-1,5 \times 10^{-2}$ & $2,6 \times 10^{-2}$ & $1,1 \times 10^{-1}$ & $1,3 \times 10^{-1}$ & $2,4 \times 10^{-1}$ & $-1,6 \times 10^{-2}$ & $1,5 \times 10^{-1}$ \\
\hline 12 & $\mathrm{n}=90$ & $6,8 \times 10^{-2}$ & $3,2 \times 10^{-3}$ & $5,1 \times 10^{-3}$ & $-2,3 \times 10^{-3}$ & $-9,8 \times 10^{-1}$ & $-4,7 \times 10^{-2}$ & $6,3 \times 10^{-2}$ & $-9,7 \times 10^{-2}$ \\
\hline \multirow[t]{3}{*}{$\begin{array}{l}\text { Edad } \\
\text { (meses) }\end{array}$} & $\underset{\mathbf{n}}{\operatorname{Valor}} \mathrm{de}$ & \multicolumn{2}{|c|}{$\begin{array}{l}\text { Disolución de } \\
\text { SO }_{4} \mathrm{Na}_{2} \text { al } 5 \%\end{array}$} & \multicolumn{2}{|c|}{$\begin{array}{c}\text { Disolución de } \\
\mathrm{SO}_{4} \mathrm{Mg} \text { al } 10 \%\end{array}$} & \multicolumn{2}{|c|}{$\begin{array}{c}\text { Disolución de } \\
\mathbf{S O}_{4}\left(\mathbf{N H}_{4}\right)_{2} \text { al } 5 \%\end{array}$} & \multicolumn{2}{|c|}{$\begin{array}{l}\text { Disolución de } \\
\text { CINH }_{4} \text { al } 5 \quad \%\end{array}$} \\
\hline & & \multicolumn{2}{|c|}{ Velocidad de disolución } & \multicolumn{2}{|c|}{ Velocidad de disolución } & \multicolumn{2}{|c|}{ Velocidad de disolución } & \multicolumn{2}{|c|}{ Velocidad de disolución } \\
\hline & & Sin carbonatar & Carbonatados & Sin carbonatar & Carbonatados & Sin carbonatar & Carbonatados & Sin carbonatar & Carbonatados \\
\hline 3 & $\mathrm{n}=60$ & $6,0 \times 10^{-2}$ & $1,2 \times 10^{-1}$ & $-1,0 \times 10^{-1}$ & $1,0 \times 10^{-1}$ & $-1,9 \times 10^{-1}$ & $-3,2 \times 10^{-1}$ & $-2,1 \times 10^{-1}$ & $1,1 \times 10^{-1}$ \\
\hline 6 & $\mathrm{n}=90$ & $-7,2 \times 10^{-2}$ & $1,1 \times 10^{-2}$ & $-1,5 \times 10^{-2}$ & $1,6 \times 10^{-1}$ & $6,7 \times 10^{-2}$ & $3,6 \times 10^{-2}$ & $1,6 \times 10^{-1}$ & $5,1 \times 10^{-2}$ \\
\hline 9 & $\mathrm{n}=90$ & $6,1 \times 10^{-1}$ & $5,2 \times 10^{-1}$ & $5,8 \times 10^{-1}$ & $-3,0 \times 10^{-2}$ & $-7,6 \times 10^{-1}$ & $-2,8 \times 10^{-2}$ & $3,7 \times 10^{-2}$ & $-4,4 \times 10^{-2}$ \\
\hline 12 & $\mathrm{n}=90$ & $4,1 \times 10^{-2}$ & $*$ & $1,5 \times 10^{-1}$ & $6,0 \times 10^{-1}$ & $4,5 \times 10^{-2}$ & $1,5 \times 10^{-2}$ & $9,4 \times 10^{-2}$ & $2,5 \times 10^{-1}$ \\
\hline
\end{tabular}

* No hay valores por estar desintegrados los prismas. 


\section{Conclusiones provisionales}

El conjunto de resultados se expresan en el cuadro 4. A partir de él se han establecido las siguientes conclusiones:

a) En agua filtrada.

Los prismas sin carbonatar están más atacados que los prismas carbonatados, en todas las edades estudiadas.

b) En disolución saturada de sulfato cálcico.

Salvo en la última edad estudiada, parecen estar más atacados los prismas carbonatados.

c) En disolución mezcla de sulfatos.

En conjunto están menos atacados los prismas carbonatados.

d) En agua de mar artificial.

Aunque hay bastantes dispersiones, tambien en conjunto están menos atacados.

e) En disolución de sulfato sódico al $5 \%$.

A partir del $6 .^{\circ}$ mes estudiado, las resistencias decrecen brusca y progresivamente. Aparecen menos atacados los prismas sin carbonatar.

f) En disolución de sulfato magnésico al $10 \%$.

Hasta la edad de 9 meses parecen más estables los prismas carbonatados. En todas las series estudiadas su resistencia a los sulfatos es prácticamente nula.

g) En disolución de sulfato amónico al $5 \%$.

En todos los casos el comportamiento de los prismas es el mismo.

h) En disolución de cloruro amónico al $5 \%$.

Siempre son algo más estables los prismas carbonatados.

\section{CONCLUSIONES FINALES}

Tanto de las experiencias llevadas a cabo, así como de la bibliografía estudiada sobre el tema de carbonatación, para que ésta pueda considerarse eficaz y para ensayos posteriores, habría que fijar los siguientes puntos (5):

- Estudio de las condiciones y duración óptimas del curado previo a la carbonatación. para mayor eficacia de ésta.

- Determinación del agua evaporable y del agua no evaporable, y estudio de su influencia en la carbonatación. 
- Determinación de la porosidad, volumen, tamaño, forma y distribución de los poros.

- Fijar las condiciones óptimas de carbonatación propiamente dicha, para que ésta sea eficaz. Para ello conviene estudiar:

a) influencia de la humedad relativa de la atmósfera de $\mathrm{CO}_{2}$ en la carbonatación, y

b) influencia de la presión y temperatura de carbonatación y determinación de los valores óptimos de ambas.

\section{ESTUDIO REALIZADO CON ESPECTROSCOPIA IR. CONCLUSIONES}

Una vez medida la resistencia a flexotracción se han tomado muestras de prismas, en las que se ha procurado eliminar al máximo la arena del mortero; posteriormente, se ha molido finamente la pasta para poderla estudiar por espectroscopía infrarroja.

Dado el gran número de espectros infrarrojos estudiados (realizados sobre 80 muestras), no se reproducen aquí. En una próxima publicación del I.E.T.c.c. se incorporarán los espectros mencionados, así como una más detallada marcha del trabajo.

Ateniéndonos a la extensión limitada que nos imponemos, vamos a resumir las conclusiones que, en forma provisional, hemos obtenido en este trabajo.

1) Una de las causas de la disminución de las resistencias mecánicas de las probetas, objeto de estudio es la formación de yeso que en forma progresiva se observa en los prismas conservados en las siguientes disoluciones: sulfato sódico, sulfato magnésico y sulfato amónico.

2) En las probetas que están introducidas en disoluciones en las que interviene el catión $\mathrm{Mg}^{2+}$ se forma progresivamente $\mathrm{Mg}(\mathrm{OH})_{2}$, que disminuye o desaparece a edades superiores.

3) La banda de absorción IR producida por el silicato tricálcico en los cementos anhidros, aparece a $930 \mathrm{~cm}^{-1}$. Con la hidratación normal del cemento se desplaza hasta, generalmente, $970-980 \mathrm{~cm}^{-1}$ (4). Sin embargo, en bastantes de los prismas estudiados, la banda correspondiente a la tobermorita ha sufrido un desplazamiento anormal hacia mayores frecuencias. Esta circunstancia va paralelamente unida a la disminución e incluso desaparición de la banda correspondiente al $\mathrm{Ca}(\mathrm{OH})_{2}$.

Esto, en nuestra opinión, nos indica una progresiva evolución de la tobermorita, que se va enriqueciendo en $\mathrm{Ca}^{2+}$. Este catión, por consiguiente, se combina con el $\mathrm{SO}_{4}{ }^{2-}$ para formar yeso, con el $\mathrm{CO}_{3}{ }^{2-}$ para formar $\mathrm{CO}_{3} \mathrm{Ca}$ y también, en ocasiones, puede entrar a formar parte de la estructura tobermorítica.

4) Lógicamente, la aparente anormalidad de la tobermorita formada ha de causar una alteración de la resistencia mecánica y, probablemente, de la durabilidad de las probetas afectadas. 
5) En los espectros IR de los prismas con mayor pérdida de resistencia mecánica hay una evolución muy clara de una banda de absorción situada hacia $540 \mathrm{~cm}^{-1}$, en el sentido de una disminución progresiva de intensidad, hasta su aparente desaparición.

En esta banda están incluidas absorciones Si-O-Al. Por consiguiente, la disminución de la banda nos indica que, al menos en parte, la tobermorita evoluciona a costa de los silicoaluminatos.

6) Hay formación de carbonato cálcico amorfo, en las muestras introducidas, en las siguientes disoluciones:

- Mezcla de $\mathrm{SO}_{4} \mathrm{Ca}$ y $\mathrm{SO}_{4} \mathrm{Mg}$.

- Agua de mar artificial.

$-\mathrm{SO}_{4} \mathrm{Na}_{2}$ al $5 \%$.

$-\mathrm{SO}_{4} \mathrm{Mg}$ al $10 \%$. (Aquí se produce también aragonito).

Hay una correlación entre la existencia del carbonato cálcico, gel, y el proceso de hidratación del cemento.

Cuando fue necesario, los resultados obtenidos por IR se comprobaron por difracción de rayos X. Queremos hacer constar aquí nuestro agradecimiento al Doctor F. TRIVIÑO por la interpretación de los difractogramas.

\section{REFERENCIAS BIBLIOGRAFICAS}

(1) Koch y Steinegger.: Zement-Kalk-Gips, 13, p. 317; 1960.

(2) Garcia de Paredes, P.: Monografía n.² 232, del Instituto Eduardo Torroja de de Construcción y del Cemento.

(3) Fernandez-Peña, O.: Cuaderno de Investigación n. ${ }^{\circ}$ 6, del I.E.T.c.c.

(4) Lehmann, H. y Dutz, H.: Tonindustrie Zeitung; p. 219, n. ${ }^{\circ}$ 10, 1959.

(5) Fernandez-Peña, O.: Comunicación presentada a la Primera reunión del Grupo de Carbonatación de la RILEM, celebrada en el CERILH, París, marzo de 1971. 